\title{
Calibration of Image Analysis Systems for Statistically Demanding QM Applications
}

\author{
Barry M Jenkins \\ Jenkins-Kwan Technology Pty. Ltd., Queensland Centre for Advanced Technologies, PO Box 883, \\ Kenmore, QLD 4069, AUSTRALIA
}

A generic framework has been developed to solve diverse industrial QM (Quantitative Microscopy) tasks by image analysis. The approach has evolved from petrography and mineralogy applications where extreme sample heterogeneity was encountered. The applications include analysis of particles (coal, iron ore, sinter, salt etc.) in the size range of 1 micron to $1 \mathrm{~mm}$, using SEM (scanning electron microscopy) and LM (light microscopy) [1-3]. The guiding principles, which can also apply to metallurgical or biological microscopy applications, will be discussed.

These applications are amongst the most statistically demanding in the QM field (a single grainmount specimen can represent a train-load of ore or a large section of an exploration area). Therefore, representative sampling was considered on a whole-of-system basis, defined as the system ergodicity [3]. All steps affecting an analysis are covered, including: physical sampling, electronic sampling, digital sampling, image sampling and the final representation of data in analytical models. The systems were designed around Carl Zeiss $\mathrm{KS}^{\mathrm{TM}}$ image analysis and microscope integration software. All SEM and LM modes of imaging can be integrated, including confocal imaging and spectroscopy. If more than one mode is required to overcome uniqueness problems, image fusion techniques may be employed to combine the complementary data [3]. Image analysis routines are then developed which include systematic calibration procedures to enhance the robustness and adaptability of the system. Key image analysis steps in the measurement routines are then parameterized (e.g. the number of erosions or dilations used in edge segmentation) to allow their adjustment during final calibration. Factors that constrain the application (e.g. microscope setup conditions) are included in a knowledge-base. Finally, an analysis management system is used to control the analysis or prompt an operator.

\section{CASE STUDY: Automated Coal Petrography}

This was a reflected-light microscopy application where primary reference data was provided by manual analyses of vitrinite reflectance (coal rank) and maceral abundances [1]. These are quality measures used routinely for setting sale contracts and for predicting performance in coke making plants and power plants. Development of the system was based on the Australian Standard AS2856 (ASTM D2798 equiv.)[5].

\section{Microscope Setup and Specimen Quality}

Multiple reflectance standards were used to set the light level, to map the light distribution across the field of view, and to calibrate camera linearity. If the grey level variance was too great, or cross referencing of reflectance values showed an error, the management system alerted the operator to adjust the illuminator or clean the standards. An analyst can work around most defects in a specimen surface. To include this flexibility in the automated system, a pre-scan of the specimen was used to assess surface quality and manage the corrective processing. The extent of scratching was measured, over-polishing was gauged from the extent of topography at pore boundaries, and the differences in 'image quality' due to different mounting resins was detected. The results were used to tune the image reconstruction processes in each analysis, and provide feedback to the sample preparation laboratory.

\section{Image Analysis}

Coal analysis required the creation of reflectance-calibrated images. Each raw image was systematically broken down into the smallest significant texture elements or component structures. This allowed separate processing of elements and permitted different reconstructions for various classification schemes, standards and definitions. Complex manipulations were performed on each image to determine the optimum processing parameters. Original images were then processed with the most succinct and simple routines. The essential steps were shading correction (to calibrate each pixel for reflectance) and topography correction (based on gray level variance within each phase). If system ergodicity was maintained, the final reconstructed image represented the true structure accurately. The total number of images (or fields) used in an analysis was controlled by the percent relative accuracy $(\% \mathrm{RA})$ required for each measured parameter. 


\section{Calibration}

The calibration routines cover system parameters, instrument setup, differences in specimen preparation, and image analysis operations. The initial system calibration was by reference to a single laboratory [7]. To test the system against a wider representation of the coal petrographer community, it was entered in the Petrographer Accreditation Programme of the International Committee for Coal and Organic Petrology (TABLE 1) [6]. In these exercises the 'right' answer is given as the group mean values. The results (based on over 50 laboratories) were used in the final calibration of the system, which improved the system's performance markedly.

TABLE 1. Assessment of (A) Vitrinite Random Reflectance Analysis and (B) Maceral Content Analysis [6]. In 2000 the system was calibrated against a single laboratory. In 2002 the system was calibrated against the group mean results of the 2000 exercise. Ranking is by UMSD (Unsigned Multiple of the Standard Deviation) and SMSD (Signed Multiple of the Standard Deviation).

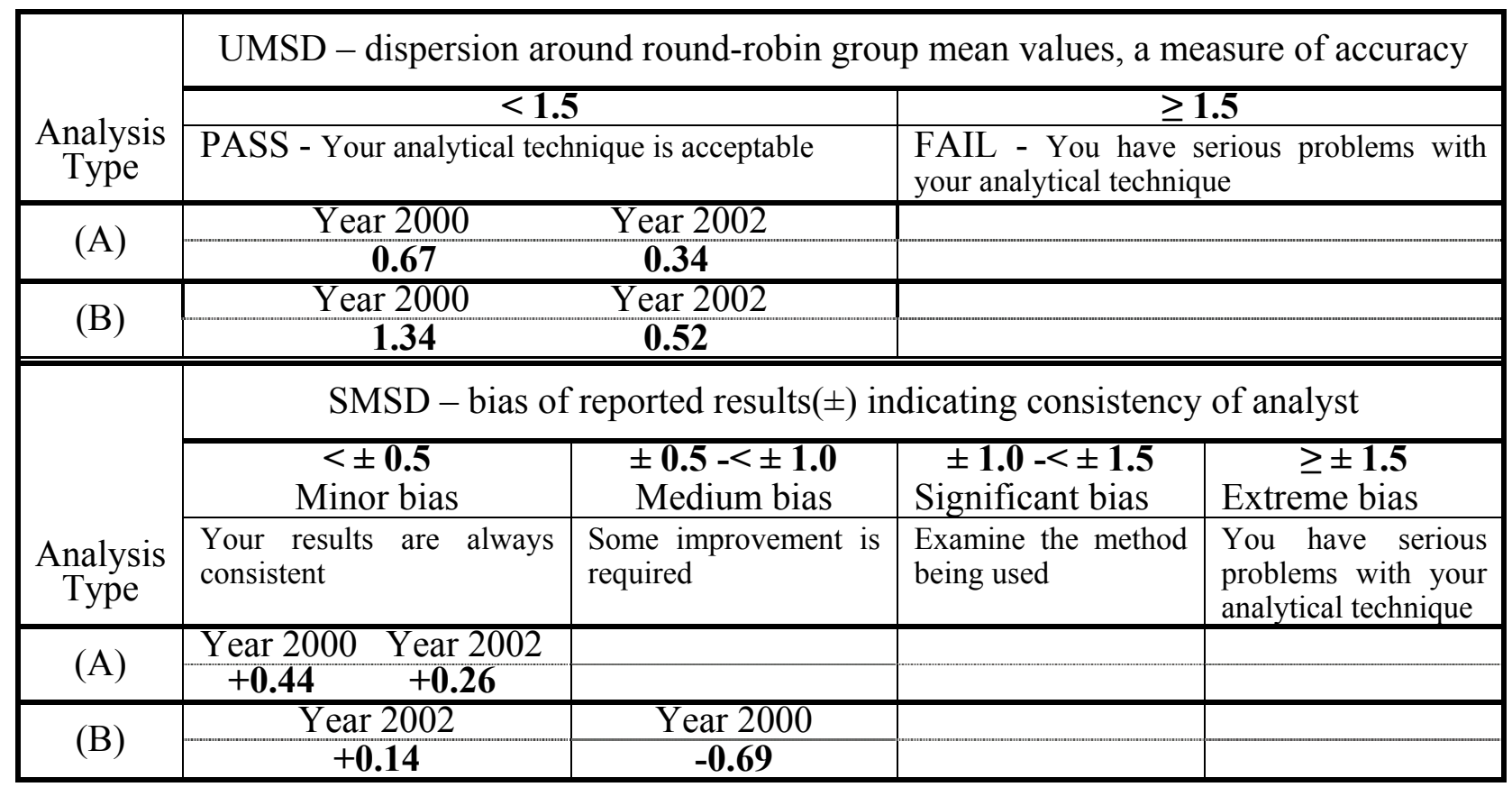

\section{Data Analysis}

Data analysis routines were executed in a spreadsheet environment. This included corrections in some measurement types for stereological bias [4].

\section{Conclusions}

The design philosophy produced a coal analysis system that met existing manual standards and provided a foundation for a range of new and unique analyses. Generally:

- the design methodology is suitable for complex characterization problems

- output can be tailored to meet different standards and classification systems

- automatic analysis of 'image quality' can improve system performance.

- management of microscope handling and sample preparation practices is important

- participation in round-robin exercises can improve system calibration.

[1] B.M. Jenkins et al., Proc. Inst. Mat. Eng. Aust (1998).

[2] B.M. Jenkins et al., (1990), 292-309, ASTM STP 1094, George F. Vander Voort, Ed., Philadelphia.

[3] B.M. Jenkins et al., in proceedings ISSN 1325 3034, (1995):401-407, 455-460

[4] G.Barbery. Mineral Liberation (1991). Quebec: Les Editions GB.

[5] The Australian Coal Research Program (ACARP) was a major sponsor of this research.

[6] The cooperation of Mr Aivars Depers and the ICCP is gratefully acknowledged.

[7] The collaboration of Mr Graham O'Brien and CSIRO is gratefully acknowledged. 